Raynauds phenomenon, peripheral neuropathy, renal involvement and thrombocytopenia, was found to be important overall for discriminating SLE patients with or without SS. SLEwSS patients constitute a subgroup of patients with SLE characterized by milder lupus: older age at death, similar rates of mortality and SLICC-ACR damage index, less renal and immunological manifestations.

Funding Source(s): None

\section{PERSISTENCE OF ANTI-SMITH ANTIBODY IS ASSOCIATED WITH DISEASE ACTIVITY IN PATIENTS WITH NEW-ONSET SYSTEMIC LUPUS ERYTHEMATOSUS}

Sung Soo Ahn*, Seung Min Jung, Yong-Beom Park. Yonsei University College of Medicine

\subsection{6/lupus-2019-Ism.122}

Background Anti-Smith ( $\mathrm{Sm}$ ) antibody is highly specific antibody for systemic lupus erythematosus (SLE). We evaluated the association between anti-Sm antibody and disease activity in patients with new-onset SLE.

Methods Patients who were repeatedly tested for anti-Sm antibody at SLE diagnosis and within 12 months were included in this study. The clinical and laboratory profiles, and systemic lupus erythematosus disease activity index (SLEDAI) were collected at the time of anti-Sm antibody test. SLEDAI and laboratory variables associated with disease activity were compared between patients with and without anti-Sm antibody.

Results Of 92 patients who were tested for anti-Sm antibody at SLE diagnosis, 67 patients were followed up for presence of anti-Sm antibody at 6 months, and 67 patients were followed up at 12 months. Although the baseline SLEDAI was comparable in SLE patients with or without anti-Sm antibody, immunologic and hematologic disorder was more common in anti-Sm positive patients. Patients who showed positive result of anti-Sm antibody at 6 and 12 months had higher SLEDAI compared to patients with negative result $(p=0.004$ and 0.002 at 6 and 12 months, respectively). The changes in anti-Sm antibody for 12 months was significantly correlated with the changes of SLEDAI $(p=0.029)$.

Conclusions Persistence of anti-Sm antibody for 12 months was associated with higher disease activity at the corresponding time. Follow-up of anti-Sm antibody can be useful to evaluate the remained disease activity in patients with new-onset SLE. Funding Source(s): None

\section{CLINICAL CHARACTERISTICS OF PATIENTS WITH SYSTEMIC LUPUS ERYTHEMATOSUS SHOWING THE FALSE POSITIVE RESULT OF SYPHILIS TEST}

Sung Soo Ahn*, Seung Min Jung, Yong-Beom Park. Yonsei University College of Medicine

10.1136/lupus-2019-|sm. 123

Background False positive result of syphilis test is a characteristic finding in patients with systemic lupus erythematosus (SLE), especially combined with antiphospholipid syndrome (APS). We evaluated the clinical characteristics in SLE patients who showed the false positive result of syphilis test.

Methods Patients who were tested for syphilis screening test at SLE diagnosis in Severance Hospital between January 2006 and December 2016 were included in this study. The baseline characteristics and clinical outcomes were compared between patients with false positive result of syphilis test and negative result of syphilis.

Results Of 145 patients included in this study, 20 (13.8\%) patients showed the false positive result of syphilis test. At SLE diagnosis, patients with negative syphilis result had higher SLE disease activity index $(5.0$ vs $8.0, \mathrm{p}<0.001)$, and were more commonly complicated with nephritis $(15.0 \%$ vs $41.6 \%$, $\mathrm{p}=0.026)$. Low disease activity, high protein level, and presence of APS antibodies were independently associated with the false positive result of syphilis test $(p=0.030,0.014$ and 0.002 , respectively). Although the thrombotic risk was significantly higher in patients with false positive syphilis result $(p=0.041)$, the overall mortality showed no difference between patients with false positive result and negative result of syphilis test.

Conclusions Clinical characteristics of SLE patients with false positive result of syphilis test showed lower disease activity at SLE diagnosis, but comparable overall survival and higher thrombotic risk.

Funding Source(s): None

\section{ANTIRIBOSOMAL P AUTOANTIBODIES TARGET THE NEURONAL-SURFACE-P-ANTIGEN (NSPA) IN KIDNEY AND LIVER CELLS}

${ }^{1}$ Marcela Bravo-Zehnder*, ${ }^{2}$ Carmen Sofia Espinoza, ${ }^{3}$ Patricia Gajardo, ${ }^{3}$ Tomás Toledo, ${ }^{3}$ Javiera Alvarez, ${ }^{3}$ Mariana Labarca, ${ }^{1}$ Loreto Massardo, ${ }^{4}$ Alfonso González. ${ }^{1}$ Centro de Biología Celular y Biomedicina (CEBICEM). Faculty of Science and Medicine, Universidad San Sebastián. Santiago, Chile; ${ }^{2}$ Pontificia Universidad Católica de Chile; ${ }^{3}$ Universidad San Sebastián; ${ }^{4}$ Centro de Biología Celular y Biomedicina. Faculty of Science and Medicine, Universidad San Sebastián. Santiago, Chile. Centro de Envejecimiento y Regeneración (CARE), Facultad de Ciencias, Pontificia Universidad Católica de Chile. Santiago, Chile

\subsection{6/lupus-2019-Ism.124}

Background Anti-ribosomal P (anti-P) antibodies have long been associated with lupus psychosis and recently with cognitive deficit in patients with systemic lupus erythematosus (SLE). We previously described a neuronal-surface-P-antigen (NSPA) that mediates anti-P pathogenic effects leading to memory deficit in mice. Clinical controversial associations of anti-P are lupus hepatitis and lupus nephritis (LN), in which the ribosomal $\mathrm{P} 0$ protein has been postulated as a cell surface anti-P target. As there is no mechanism explaining for the presence of $\mathrm{P} 0$ at the cell surface, we assess whether NSPA might be the anti-P cell surface target in liver and kidney cells.

Methods NSPA expression: i) RT-PCR and immunoblot in liver HepG2 and kidney HK-2 cell lines and liver and kidney tissues from C57wt and transgenic C57NSPA/KO (LacZ gene instead NSPA gene) mice; ii) ß-galactosidase histochemistry as a marker of NSPA expression in liver and kidney slices from a ZZEF-1/lac Z knock in mice; iii) Cell surface biotinylation and surface immuno-capture in combination with metabolic labeling. Functional assays: iv) Internalization assays with I125anti-P; v) Indirect immunofluorescence of activated caspase-3 and Hoechst staining of apoptotic nuclei on HK-2 treated with rabbit anti-P for 24 hour.

Results NSPA is expressed in hepatocytes, in proximal epithelium tubule renal cells and in some collecting tubules. HK-2 and HepG2 express NSPA, but not P0 at the cell surface. Anti-P bind NSPA and become internalized in a time and concentration dependent manner. Anti-P induced HK-2 apoptosis in vitro assessed by caspase- 3 activation. 